\title{
Optimisation de données bathymétriques à l'aide de "SURFER": Application à l'historique des plages d'Anglet
}

\author{
Philippe Maron ${ }^{(1)}$, Didier Rihouey ${ }^{(2)}$ \\ ${ }^{(1)}$ Maître de Conférences, LaSAGeC - Génie Côtier, Université de Pau \\ et des Pays de L'Adour, I.S.A. B.T.P., 1 allée du Parc Montaury, \\ 64600 ANGLET, France, E-mail: philippe.maron@univ-pau.fr, \\ Tél: 05.59.57.70.16. \\ ${ }^{(2)}$ Doctorant LaSAGeC - Génie Côtier, Université de Pau et des Pays \\ de L'Adour, I.S.A. B.T.P., 1 allée du Parc Montaury, 64600 ANGLET, \\ France, E-mail: didier.rihouey@univ-pau.fr,.Tél: 05.59.57.70.42. \\ Résumé : \\ L'analyse des données contenues sur des cartes bathymétriques est possible \\ à condition de se poser un certain nombre de questions sur les méthodes de \\ récupération des données sous forme numérique, puis sur la modélisation de ces \\ données à l'aide d'un logiciel GIS (Geographic Information System). Cet article \\ montre que contrairement à l'idée première, il convient de numériser les points de \\ mesures et non la position des isobathes. Les importantes variations, engendrées \\ par le nombre de points de mesures, sont également mises en évidence. Enfin la \\ comparaison des différentes méthodes de modélisation proposées par le logiciel \\ "Surfer 7" de Golden Software, a montré que les algorithmes "Kriging" et \\ "Minimum Curvature" donnaient les meilleurs résultats sur le site d'Anglet.
}

\begin{abstract}
:
A study of beaches evolution along the Anglet coast line is necessary to understand the hydrodynamic phenomena responsible for their erosion. This study is based on 22 bathymetric maps, surveyed between 1971 and 1996. Analysis of this data is possible if we wonder several questions about methods of data acquisition in a numerical format, and the data modelling with GIS software (Geographic Information System). This paper shows that it is necessary to digitalize measured points and not bottom contours. The important variations due to the number of measured points are also underlined. Finally, the comparison of different modelling methods suggested that "Kriging" and "Minimum Curvature" give the best results for data available in Anglet.
\end{abstract}

\section{Introduction}

Pour acquérir une meilleure connaissance des phénoménes d'érosion des plages de la Côte Basque, la Communauté d'Agglomération du BAB (BayonneAnglet-Biarritz) nous a chargé du suivi du littoral des plages d'Anglet. Les plages d'Anglet s'étendent sur près de 4,5 km entre l'embouchure de l'Adour au Nord, et 
le Cap St Martin au Sud, figure 1. Une bonne connaissance du site et de son historique est indispensable à la compréhension des différents phénomènes qui seront étudiés par la suite. Les différentes données et rapports disponibles ont été collectés auprès de la D.D.E. Maritime et de la Sogreah. Un travail préliminaire à l'exploitation des données bathymétriques est présenté ici.

Cette étude s'appuie sur les mesures de bathymétrie réalisées entre 1971 et 1996. Vingt deux cartes différentes ont été collectées auprès de la DDE maritime et de la Sogreah. Les données numériques n'étant pas disponibles, il a été nécessaire de les numériser. La transcription des cartes sous forme numérique suscite un certain nombre de questions: Comment exploiter des tracés manuels ou automatiques pour obtenir des données sous forme de fichiers $(\mathrm{x}, \mathrm{y}, \mathrm{z})$ ? Quelle est l'influence du nombre de points numérisés sur le résultat final? Quelle(s) méthode(s) de numérisation utiliser? Quelle(s) méthode(s) d'interpolation permettra d'obtenir une représentation satisfaisante?...

Cet article présente une tentative de réponses à travers l'étude de l'influence du nombre de points numérisés, la comparaison des méthodes de numérisation et enfin la comparaison des résultats des différentes méthodes de modélisation proposées par le logiciel "Surfer 7" de Golden Software.

\section{Moyens de mise en ouvre}

\subsection{Méthodes de numérisation des cartes bathymétriques}

Lors de la numérisation d'une carte bathymétrique, la méthode qui parait la plus simple et la plus rapide consiste à suivre le tracé des isobathes. Cette méthode est couramment utilisée et deux applications de celle-ci sont proposées.

La méthode $\mathrm{n}^{\circ} 1$ est une méthode manuelle qui consiste à mesurer, à l'aide d'une règle graduée, les positions des isobathes sur des droites parallèles à l'un des axes de coordonnées Lambert III, puis à rentrer ces valeurs dans un logiciel qui fournit les coordonnées des points. La méthode $\mathrm{n}^{\circ} 2$, utilisée par R. Polette [1] consiste à numériser les points à l'aide du logiciel "MapInfo" sur une image de la carte. Sur les cartes anciennes, les tracés des isobathes ont souvent été effectués à la main. La précision est donc très aléatoire et dépend fortement de l'opérateur. Ces deux méthodes sont basées sur le principe de repérer les isobathes tracées sur la carte d'origine. Les erreurs du tracé d'origine sont donc reprises et accentuées par les erreurs propres à chacune des deux méthodes. La seconde méthode permet d'obtenir rapidement beaucoup plus de points que la première.

Ayant eu l'opportunité d'obtenir une image de la carte de mars 1980, celle-ci a été numérisée en repérant les positions des points mesurés lors de la campagne (points positionnés par le séparateur décimal de la cote en Z). Ces mesures ont été effectuées le long de lignes perpendiculaires au rivage et distantes d'environ $100 \mathrm{~m}$. Un fichier, théoriquement identique à celui d'origine, aux erreurs de pointage de numérisation près, est ainsi obtenu. Cette troisième méthode fournit un fichier qui pourra donc servir de référence lors de la comparaison des méthodes précédentes. 


\subsection{Principes de fonctionnement des logiciels GIS}

La modélisation de surface est un processus mathématique par lequel une surface continue est interpolée à partir d'un jeu de données $(\mathrm{x}, \mathrm{y}, \mathrm{z})$ distribuées aléatoirement. Le résultat de cet interpolation fournit une donnée structurée appelée "grille". Cette surface interpolée est artificielle car ses points, bien que calculés à partir des données de départ ne sont pas confondus avec ceux-ci. Toutefois l'utilisation de ces grilles est indispensable pour pouvoir tracer des isocontours, calculer des volumes ou des surfaces, générer des coupes dans différentes directions, ... La précision de la grille obtenue et donc de la surface interpolée dépend des données de départ (nombre, répartition, ...) mais également de l'algorithme de calcul des points de la grille. Dans le cas des bathymétries, Gorman [2] indique que les procédures convergentes fournissent les résultats les plus satisfaisants. Ces algorithmes affectent, aux données voisines du point à calculer, des poids de façon à ce qu'un point proche ait plus d'influence qu'un point éloigné. Plusieurs itérations sont effectuées pour arriver au résultat final.

\subsection{Méthode d'interpolation des données}

Différentes méthodes d'interpolation ont été comparées. Une brève description des principes de chacune de ces méthodes est proposée ci dessous.

La méthode Puissance Inverse des Distances (Inverse Distance to a Power; IDP) est un interpolateur basé sur des calculs barycentriques utilisant les points voisins et leur affectant un poids, fonction inverse d'une puissance de la distance et d'un coefficient de lissage. C'est un interpolateur exact; une de ses caractéristiques est la génération de forme concentrique, "Eil de bœuf", autour des valeurs à interpoler.

La méthode de Proche Voisinage (Nearest Neighbor, NN) assigne à chaque nœud de la grille, la valeur du point le plus proche du nœud. Cette méthode est parfaitement adaptée dans les cas de répartition régulière des points de mesure, ou lorsque les points de mesure sont déjà répartis sur une grille, mais qu'il manque des données.

La méthode Krigeage (Kriging; K) est une méthode de grille géostatistique très utilisée dans différents domaines (géophysique, géologie, traitement d'images, $\mathrm{CAO}$ et $\mathrm{CFAO}, \ldots)$. Le Krigeage utilise des variogrammes : courbes donnant le poids à affecter aux points de données en fonction de leurs distances par rapport au point à interpoler. Cette méthode permet donc de respecter le type de topographie de la zone à étudier. De plus les variogrammes pouvant être différents suivant les directions, il est possible d'introduire un caractère d'anisotropie à la surface à modéliser. La méthode fonctionne par itérations successives afin de minimiser les erreurs d'interpolation.

la méthode des Réseaux Radiaux de la Fonction de Base (Radial Basis Function; RBF) regroupe plusieurs méthodes proches du Krigeage. Ce sont des interpolateurs exacts. La fonction de base, ici une multiquadratique inverse, définit le jeu de poids à appliquer aux données afin d'interpoler la grille.

Enfin la méthode de Courbure Minimale (Minimal Curvature, MC) interpole une surface, générée à partir de fines surfaces linéairement élastiques passant par tous les points de données avec des courbures minimales. Elle génère 
donc une surface la plus lisse possible tout en se rapprochant au maximum des données. Ce n'est pas un interpolateur exact.

Pour plus de renseignements sur ces différentes méthodes, le lecteur est invité à consulter les références [5], ainsi que [6] à [9].

\subsection{Zone d'étude et projection}

La zone d'étude se situe, en coordonnées Lambert III, dans une surface définie entre $284500<x<288500$ et $140250<y<145000$. Pour des raisons pratiques, nous avons choisi d'utiliser un repère local dont l'axe $X$ est sensiblement parallèle au littoral. Nous avons donc effectué un changement de repère en effectuant une rotation de 1,047 radians et en prenant pour origine le point $\mathrm{O}$, de coordonnées Lambert III $(285800,140100)$, situé en bordure de la falaise du cap St-Martin. Afin d'obtenir des données dans toute la zone d'étude, celle-ci a été limitée entre $200<\mathrm{X}<5100$ et $-200<\mathrm{Y}<1500$.

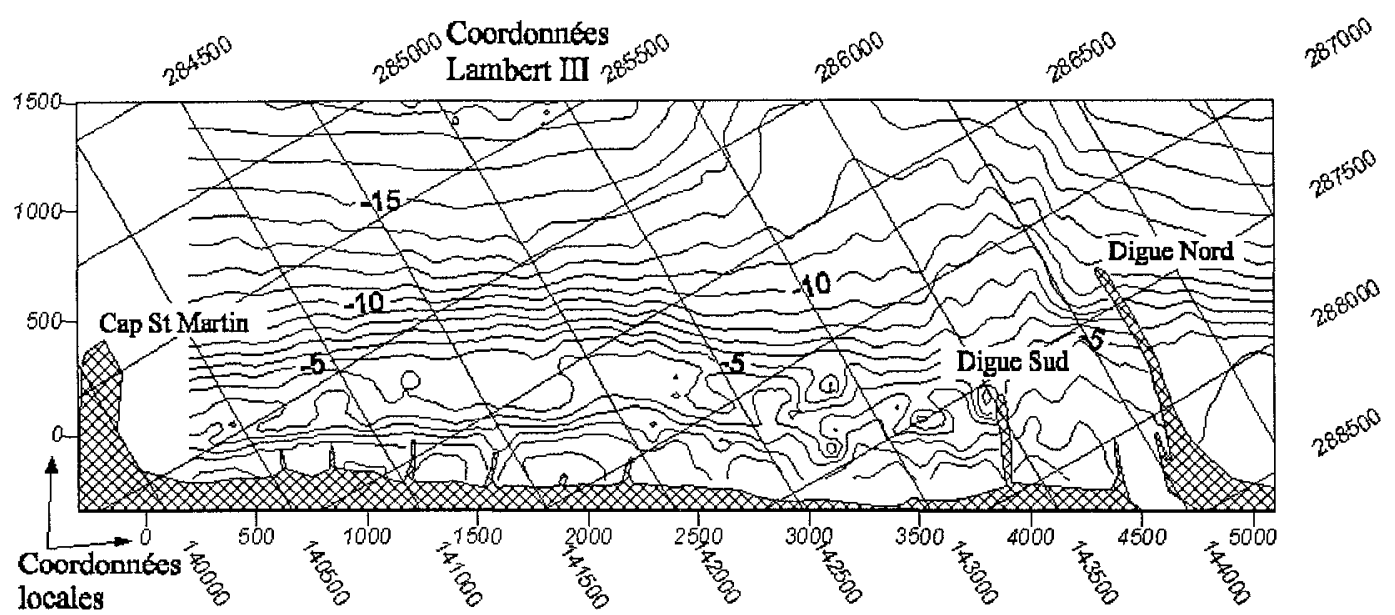

Fig. 1: Les systèmes de coordonnées Lambert III (en oblique) et locale

Fig. 1: Local and Lambert III systems of coordinates

\section{3.- Comparaison des méthodes de numérisation}

Nous avons comparé les trois méthodes de numérisation à l'aide du fichier de mars 1980. Le fichier obtenu par la méthode $n^{\circ} 1$ contient 1337 points, celui obtenue par la méthode $n^{\circ} 2,7017$ points, et celui obtenu par la méthode $n^{\circ} 3,2412$ points. Ces fichiers ont été traités par la méthode de "Krigeage" afin d'obtenir des grilles de pas carrés de $25 \mathrm{~m}$ de coté. La méthode de "Krigeage" est celle proposée par défaut dans le logiciel "Surfer".

\subsection{Calcul des résidus}

Une fois les grilles obtenues, les valeurs de profondeur $Z$ données par celles ci et celles du fichier de référence (méthode $n^{\circ} 3$ ), sont comparées en calculant les résidus $Z_{\text {res }}$ :

$$
Z_{\text {res }}=Z_{\text {dat }}-Z_{\text {grd }}
$$

avec $Z_{d a t}$, la valeur de la donnée de référence et $Z_{g r d}$, la valeur calculée de la grille. 
Différents tests classiques ont été effectués. Les résultats obtenus sont présentés dans le tableau 1 . ci dessous:

\begin{tabular}{|l|r|r|r|}
\hline & Méthode 1 & Méthode 2 & Méthode 3 \\
\hline Somme des carrés des résidus $\left(\mathrm{m}^{2}\right)$ & 205.12909 & 210.43959 & 13.68281 \\
\hline Valeur moyenne des résidus $(\mathrm{m})$ & -0.07673 & -0.10386 & 0.00002 \\
\hline Ecart type des résidus $(\mathrm{m})$ & 0.29154 & 0.27664 & 0.07535 \\
\hline
\end{tabular}

Tab.1: Comparaison des méthodes de numérisation: calcul sur les résidus

Tab.1: Comparison of methods of digitalization: calculation on residus

Les deux premières méthodes donnent des résultats sensiblement similaires, mais très différents de ceux obtenus par la troisième méthode. La numérisation par relevés des isobathes ne semble donc pas satisfaisante du point de vue de ce critère de comparaison. De plus il semble que le nombre de points relevés ne modifie pas la précision de l'interpolation.

\subsection{Calcul sur les volumes}

Les volumes compris entre les surfaces interpolées et le plan $\mathrm{P}$ passant par la cote $Z=0 \mathrm{~m}$ pour $Y=-200 \mathrm{~m}$ et la cote $Z=-19 \mathrm{~m}$ pour $Y=1500 \mathrm{~m}$ ont été évalués par la méthode de "règle trapézoïdale" (Trapezoidal Rule). Ce plan $\mathrm{P}$ a été choisi de façon à minimiser les valeurs des volumes et donc permettre des comparaisons plus représentatives. Le calcul du volume est effectué numériquement en commençant par intégrer par rapport à $X$ afin d'obtenir l'aire comprise sous la ligne, puis à intégrer par rapport à $\mathrm{Y}$ afin d'obtenir le volume. Les formules d'intégration sont:

$$
\begin{aligned}
& A_{i}=\frac{\Delta X}{2} \cdot\left(Z_{i, 1}+2 \cdot Z_{i, 2}+2 \cdot Z_{i, 3}+\ldots+2 \cdot Z_{i, n-1}+Z_{i, n}\right) \\
& \text { Volume } \approx \frac{\Delta Y}{2} \cdot\left(A_{1}+2 \cdot A_{2}+2 \cdot A_{3}+\ldots+A_{n-1}+A_{n}\right)
\end{aligned}
$$

avec $\Delta \mathrm{X}$ le pas des colonnes, $\Delta \mathrm{Y}$ le pas des lignes, $Z_{\mathrm{i}, \mathrm{j}}$ la valeur de la profondeur à la ligne $\mathrm{i}$ et la colonne $\mathrm{j}$. Pour plus d'informations sur ces méthodes de calcul des volumes, le lecteur pourra consulter les références [5] et [10].

Les résultats obtenus sont présentés dans le tableau 2 ci dessous.

\begin{tabular}{|l|c|c|c|}
\hline & Méthode 1 & Méthode 2 & Méthode 3 \\
\hline Volume $\left(\mathrm{m}^{3}\right)$ & 4735955 & 5725138 & 4253675 \\
\hline Erreur relative $(\%)$ & 11.34 & 34.59 & 0 \\
\hline
\end{tabular}

Tab. 2 : Comparaison des calculs de volumes sur les fichiers de mars 1980

Tab. 2 : Comparison between volumes calculations on 1980 march data

On constate que l'erreur sur le calcul des volumes, relative à celui obtenu par la troisième méthode, est de l'ordre de 35\% dans la seconde méthode et de l'ordre de $11 \%$ pour la première. Cette différence peut s'expliquer par les erreurs dues à la numérisation des cartes, mais peut-être également à l'opérateur ayant saisi les points. Ce résultat peut toutefois paraitre surprenant si l'on tient compte que le fichier donnant l'erreur la plus importante contient environ cinq fois plus de points. Cette remarque nous amène naturellement à nous poser la question de 
l'influence du nombre de points de données sur le résultat obtenu. Cette question sera débattue dans le paragraphe 4 . Néanmoins, du point de vue du critère de calcul du volume, les deux méthodes obtiennent des résultats éloignés de celui de la méthode de référence.

\subsection{Tracé des isobathes}

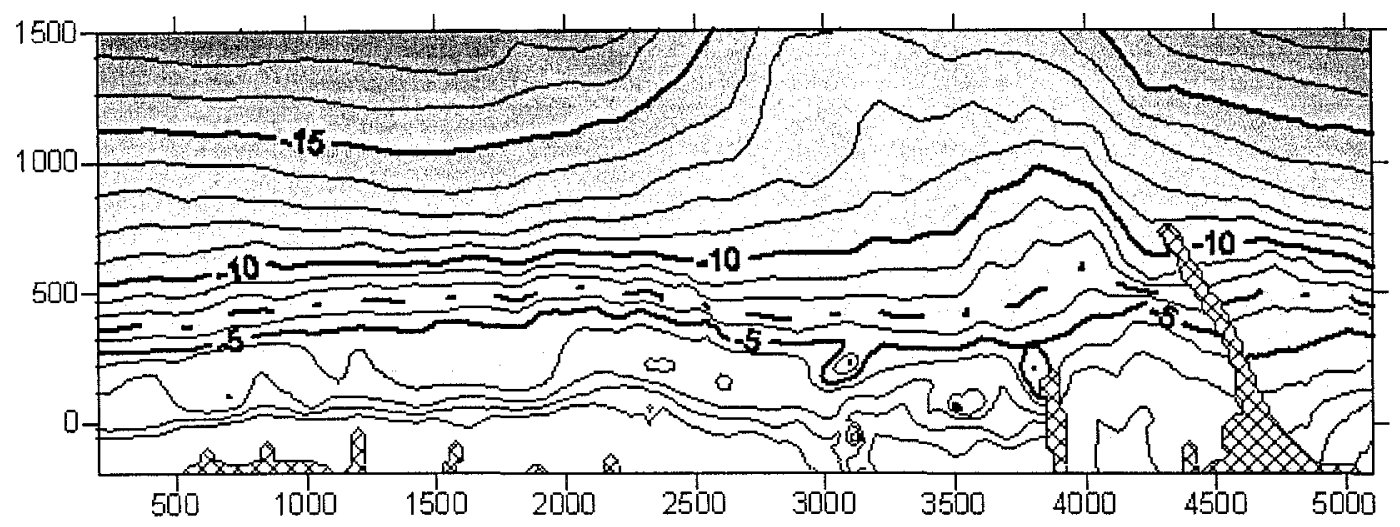

Fig. 2 : Tracé des isobathes à l'aide du fichier numérisé par la méthode $\mathrm{n}^{\circ} 1$

Fig. 2 : Contoured bathymetry for digitalizing method number 1



Fig. 3 : Tracé des isobathes à l'aide du fichier numérisé par la méthode ${ }^{\circ} 2$

Fig. 3 : Contoured bathymetry for digitalizing method number 2

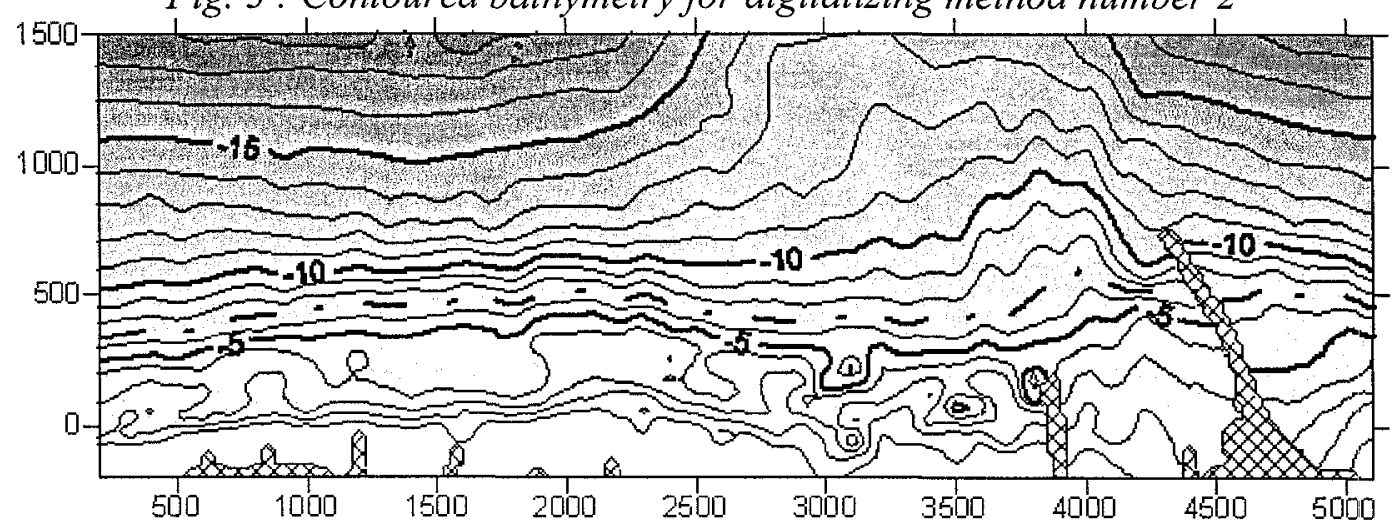

Fig. 4 : Tracé des isobathes à l'aide du fichier numérisé par la méthode $n^{\circ} 3$

Fig. 4 : Contoured bathymetry for digitalizing method number 3 
En comparant les tracés d'isobathes obtenus par les trois méthodes, figures 2,3 et 4 , la constatation qui s'impose est que les tracés sont très proches pour les deux premières méthodes. Pour des profondeurs supérieures à $Z=-5 \mathrm{~m}$, il est même difficile de faire des différences entre les trois méthodes. Toutefois, certains détails au niveau des isobathes $Z \leq-5 \mathrm{~m}$ apparaissent mieux dans la méthode $\mathrm{n}^{\circ} 3$.

Bien que ce soit la première idée qui vienne à l'esprit, la numérisation des isobathes ne donnent pas des résultats satisfaisants, même avec un grand nombre de points relevés. En effet des erreurs ont été effectuées lors du tracé initial de ces courbes. Ces erreurs sont alors reprises et éventuellement accentuées par les erreurs dues à la numérisation. De plus dans le cas de cartes anciennes, les tracés manuels dépendaient beaucoup de l'opérateur. Ces erreurs successives conduisent à des différences importantes, ici de l'ordre de $35 \%$ sur le calcul du volume.

\section{$\underline{\text { 4. Influence du nombre de points numérisés et utilisés lors du calcul de grille }}$}

Quelle que soit la méthode employée, la numérisation des données est une opération longue à mettre en œuvre. Ceci nous amène naturellement à tenter de définir un nombre optimal de points à saisir.

La carte numérisée de mars 1980 (2412 points) correspond à une campagne de mesures durant laquelle des mesures ont été effectuées environ tous les $25 \mathrm{~m}$ le long de lignes perpendiculaires à la côte et distantes d'environ $100 \mathrm{~m}$. Des fichiers ont été recréés afin de modéliser des mesures plus espacées du point de vue transversal ou longitudinal.

Les écarts testés sur les mesures transversales sont de $50 \mathrm{~m}, 75 \mathrm{~m}$ et $100 \mathrm{~m}$, permettant d'obtenir respectivement des fichiers contenant 1156 points, 804 points et 603 points. Les écarts longitudinaux entre les transversales de mesures sont de $200 \mathrm{~m}, 300 \mathrm{~m}$, et $400 \mathrm{~m}$, soit respectivement des fichiers de 1505 points, 839 points, et 771 points.

Il est évident que les observations effectuées dans cette partie de l'étude s'appliquent également au nombre de points mesurés lors d'un relevé bathymétrique.

\section{1.- Calcul sur les résidus}

Les résultats de différents tests classiques sur les résidus sont présentés dans les tableaux 3 et 4 ci dessous.

L'erreur relative $\mathrm{E}_{\alpha}$ sur la valeur $\alpha$ utilisée ci dessous est définie par:

$$
E \alpha=\frac{100 *\left(\alpha-\alpha_{\text {ref }}\right)}{\alpha_{\text {ref }}}
$$
référence.

$\alpha$ représentant la valeur de la variable et $\alpha_{\text {ref }}$ la valeur de la variable de

Ces tableaux montrent que la perte d'informations due à une plus grande distance entre les points de mesures engendre des erreurs très importantes du point de vue du critère résidu 


\begin{tabular}{|l|c|c|c|c|}
\hline \multicolumn{1}{|c|}{ Valeurs transversales } & $\mathbf{2 5} \mathbf{~ m}$ & $\mathbf{5 0} \mathbf{~ m}$ & $\mathbf{7 5} \mathbf{~ m}$ & \multicolumn{1}{c|}{$\mathbf{1 0 0} \mathbf{~ m}$} \\
\hline Somme des carrés des résidus & 13.37587 & 43.39965 & 82.57243 & 114.78511 \\
\hline Valeur moyenne des résidus & -0.00010 & -0.00185 & 0.00145 & 0.00032 \\
\hline Ecart type des résidus & 0.07450 & 0.13418 & 0.18510 & 0.21824 \\
\hline \multicolumn{1}{|c|}{ Valeurs longitudinales } & $\mathbf{1 0 0} \mathbf{~ m}$ & $\mathbf{2 0 0} \mathbf{~ m}$ & $\mathbf{3 0 0} \mathbf{~ m}$ & $\mathbf{4 0 0} \mathbf{~ m}$ \\
\hline Somme des carrés des résidus & 13.37587 & 221.99834 & 484.73658 & 520.60873 \\
\hline Valeur moyenne des résidus & -0.00010 & -0.03372 & 0.00838 & 0.11012 \\
\hline Ecart type des résidus & 0.07450 & 0.30163 & 0.44840 & 0.45154 \\
\hline
\end{tabular}

Tab. 3: Influence de la périodicité des mesures sur des axes transversaux et longitudinaux Tab. 3: Influence of spacing data along transversal and longitudinal axis

\begin{tabular}{|l|c|c|c|}
\hline \multicolumn{1}{|c|}{ Erreurs transversales } & $\mathbf{5 0 ~} \mathbf{~}$ & $\mathbf{7 5} \mathbf{~ m}$ & $\mathbf{1 0 0} \mathbf{~ m}$ \\
\hline Somme des carrés des résidus & 224.46 & 517.32 & $\mathbf{7 5 8 . 1 5}$ \\
\hline Valeur moyenne des résidus & 1750.00 & -1550.00 & -420.00 \\
\hline Ecart type des résidus & 80.11 & 148.46 & 192.94 \\
\hline \multicolumn{1}{|c|}{ Erreurs longitudinales } & $\mathbf{2 0 0} \mathbf{~ m}$ & $\mathbf{3 0 0} \mathbf{~ m}$ & $\mathbf{4 0 0} \mathbf{~ m}$ \\
\hline Somme des carrés des résidus & 1559.69 & 3523.96 & 3792.15 \\
\hline Valeur moyenne des résidus & 33620.00 & -8480.00 & -110220.00 \\
\hline Ecart type des résidus & 304.87 & 501.88 & 506.09 \\
\hline
\end{tabular}

Tab. 4: Erreurs relatives aux mesures transversales et longitudinales (\%) Tab. 4: Relative transversal and longitudinal errors (\%)

\subsection{Calcul sur les volumes}

Les volumes relatifs au plan $\mathrm{P}$ défini au paragraphe 3.2. ainsi que les erreurs relatives aux mesures tous les $25 \mathrm{~m}$ sont présentés dans le tableau 5:

\begin{tabular}{|l|c|c|c|c|c|}
\hline Fréquence transversale & $\mathbf{2 5} \mathbf{~ m}$ & $\mathbf{5 0} \mathbf{~ m}$ & $\mathbf{7 5} \mathbf{~ m}$ & $\mathbf{1 0 0} \mathbf{~ m}$ \\
\hline Volumes $\left(\mathrm{m}^{3}\right)$ & 4002562 & 4100214 & 4081786 & 4081180 \\
\hline Erreur relative $(\%)$ & 0 & 2.44 & \multicolumn{2}{|c|}{1.98} & 1.96 \\
\hline Fréquence longitudinale & $\mathbf{1 0 0} \mathbf{~ m}$ & $\mathbf{2 0 0} \mathbf{~ m}$ & $\mathbf{3 0 0} \mathbf{~ m}$ & $\mathbf{4 0 0} \mathbf{~ m}$ & $\mathbf{5 0 0} \mathbf{~ m}$ \\
\hline Volumes $\left(\mathrm{m}^{3}\right)$ & 4002562 & 4160525 & 3767217 & 2831607 & 4191192 \\
\hline Erreur relative $(\%)$ & 0 & 3.95 & -5.88 & -29.26 & 4.71 \\
\hline
\end{tabular}

Tab. 5: Influence des périodicités transversales et longitudinales sur le volume Tab. 5: Influence of spacing data on volume calculations

En ce qui concerne la fréquence transversale, les erreurs relatives sur les calculs de volume sont toutes inférieures à $2,5 \%$. Le nombre de points mesurés n'a donc pas une grosse influence sur le calcul du volume global par rapport au plan P. Par contre dans le cas de l'augmentation de l'écart entre les lignes de mesures, l'erreur peut atteindre des valeurs très importantes, de l'ordre de $30 \%$ pour un écart de $400 \mathrm{~m}$. 


\section{5- Choix d'une méthode de modélisation des surfaces}

L'objectif de cette partie est de déterminer quelle méthode il convient d'utiliser pour modéliser les surfaces. Le logiciel "Surfer" propose neuf interpolateurs différents dont cinq seulement, décrits au paragraphe 2.3. permettent d'intégrer la présence de digues ou de trait de côte.

\subsection{Calcul des résidus}

Le fichier de référence de mars 1980 a été modélisé en utilisant ces cinq méthodes et en faisant varier le pas de la grille de modélisation. Les différents pas utilisés vont de 25 à 200 par $25 \mathrm{~m}$. les valeurs de profondeur $Z$ données par les grilles ont ensuite été comparées à celles du fichier de départ, en calculant les résidus.

Enfin les sommes des carrés des résidus, ainsi que les erreurs sur ces sommes, relatives à celles obtenues pour la méthode $\mathrm{K}$ ont été calculées.

Les résultats obtenus sont données dans les tableaux 9 et 10 et la figure 5 cidessous.

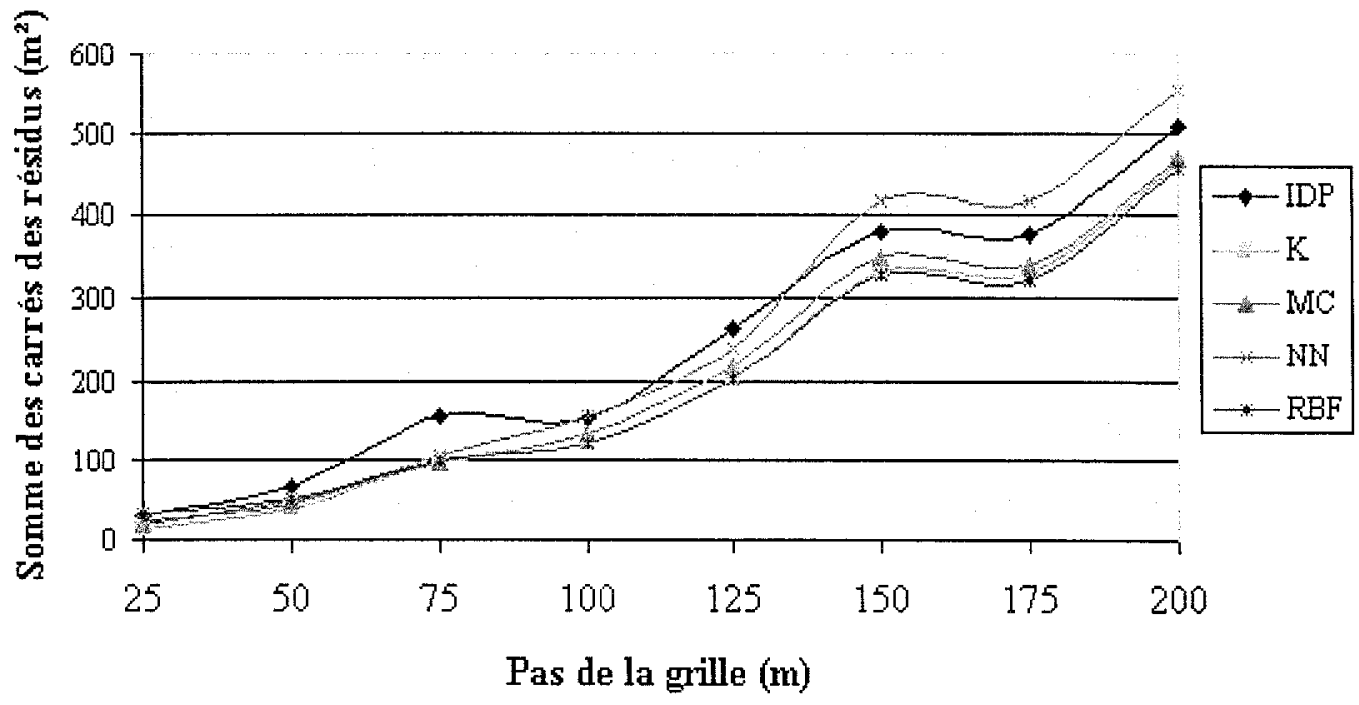

Fig. 5: Sommes des carrés des résidus obtenues par méthode et par pas de grille Fig. 5: Sum of residus square obtained for each grid methods and grid spacing

\begin{tabular}{|c|c|c|c|c|c|c|c|c|}
\hline Pas & $\mathbf{2 5}$ & $\mathbf{5 0}$ & $\mathbf{7 5}$ & $\mathbf{1 0 0}$ & $\mathbf{1 2 5}$ & $\mathbf{1 5 0}$ & $\mathbf{1 7 5}$ & $\mathbf{2 0 0}$ \\
\hline RBF & 139.36 & 36.34 & 4.20 & 0.17 & -0.28 & -1.14 & -2.89 & -1.17 \\
\hline K & 0 & 0 & 0 & 0 & 0 & 0 & 0 & 0 \\
\hline MC & 39.39 & 27.07 & 0.79 & 8.75 & 8.08 & 4.94 & 2.36 & 1.34 \\
\hline IDP & 123.84 & 77.28 & 60.69 & 24.80 & 30.01 & 14.61 & 13.29 & 9.95 \\
\hline NN & 79.95 & 20.07 & 8.50 & 25.03 & 18.29 & 25.10 & 25.55 & 20.12 \\
\hline
\end{tabular}

Tab. 10: Erreur relative à la méthode $\mathrm{K}$ avec un pas de $100 \mathrm{~m}(\%)$

Tab. 10: Relative error to $\mathrm{K}$ method with $100 \mathrm{~m}$ grid spacing (\%)

Le fichier d'origine correspond à un pas de l'ordre de $100 \mathrm{~m}$, il semble donc logique de commencer par examiner les résultats obtenus pour ce pas de grille. 
Les méthodes donnant les meilleurs résultats sont les méthodes $\mathrm{K}$ et $\mathrm{RBF}$, la méthode MC donne un résultat proche, mais sensiblement moins bon. Les deux autres méthodes obtiennent des résultats plus éloignés du fichier de référence.

Si on diminue le pas de grille afin d'essayer d'obtenir un maillage plus fin, la précision augmente. La méthode $\mathrm{K}$ donne toujours les meilleurs résultats alors que l'erreur relative de la méthode RBF augmente. Par contre pour des pas de grille plus grand, ces deux méthodes continuent de donner des résultats équivalents, meilleurs que pour les trois autres méthodes.

\subsection{Tracé des isobathes}

Les tracés des isobathes pour un pas de grille de $25 \mathrm{~m}$, obtenus par les différentes méthodes sont comparés à celui obtenu par la méthode de Krigeage.

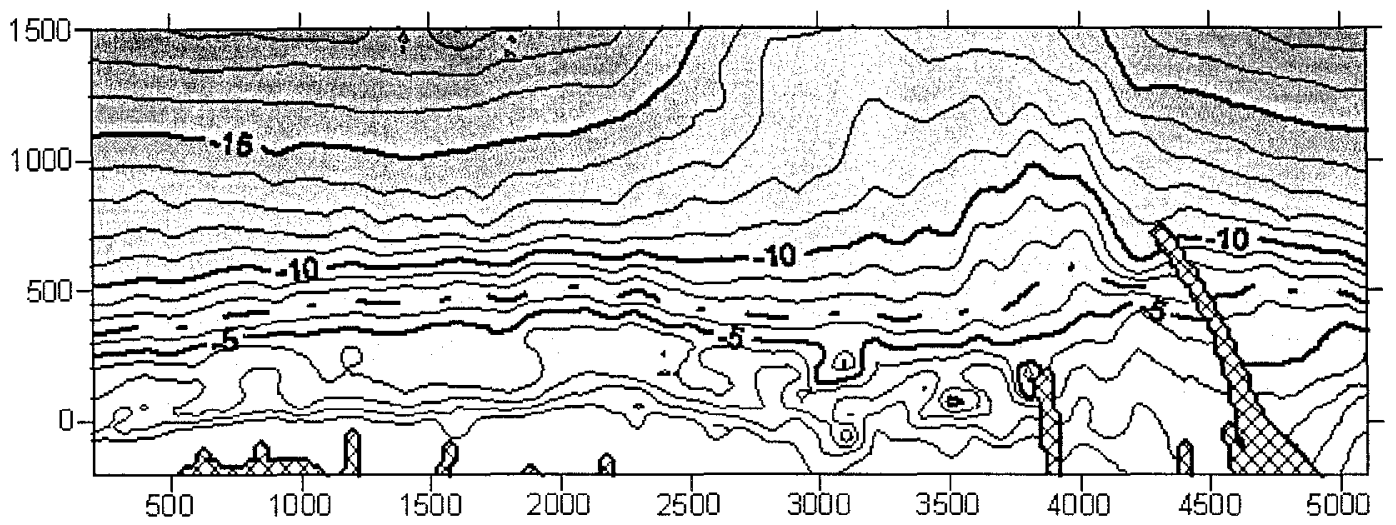

Fig. 6: Isobathes de 1980-03 par la méthode $\mathrm{K}$ avec un pas de grille de $25 \mathrm{~m}$

Fig. 6: Contoured bathymetry from 1980-03 for Kriging with $25 \mathrm{~m}$ grid spacing

Les tracés obtenus par la méthode NN présentent des profils crénelés qui ne sont pas représentatifs de la réalité. Cette méthode n'est donc pas adaptée à notre problème; en effet la répartition des points de mesure n'est pas régulière.

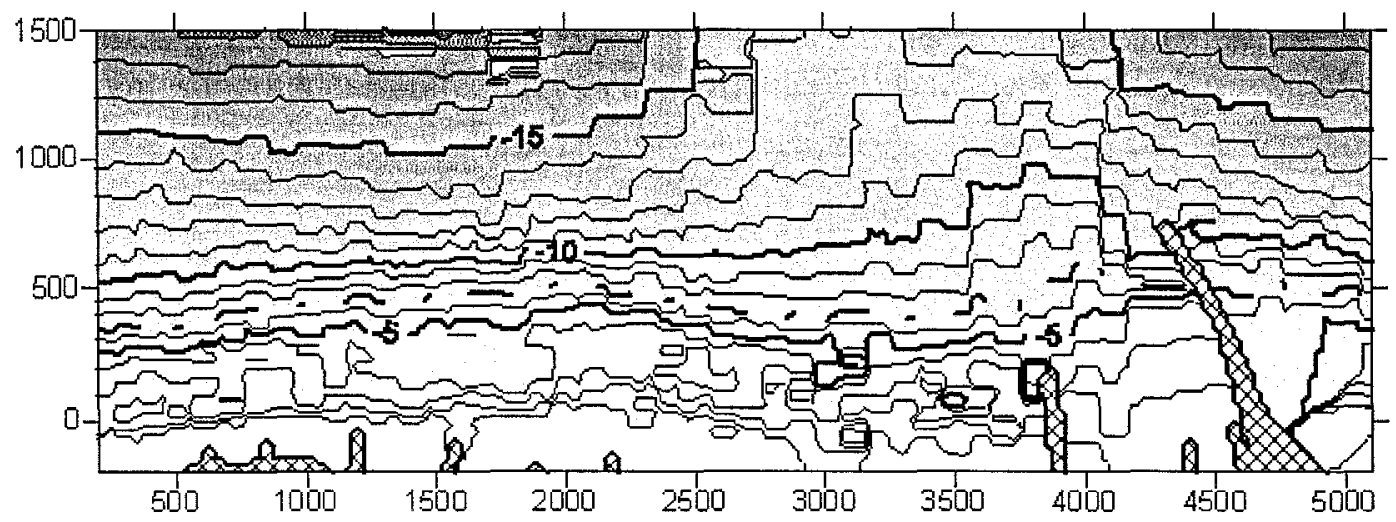

Fig. 7: Isobathes de 1980-03 par la méthode NN avec un pas de grille de $25 \mathrm{~m}$

Fig. 7: Contoured bathymetry from 1980-03 for Nearest Neighbor with $25 \mathrm{~m}$ grid spacing

La méthode IDP, présente des contours à forte tendance concentrique autour des données. Cette particularité est illustrée par l'agrandissement d'une zone située en $X$ de l'ordre de $2500 \mathrm{~m}$, figure $8-\mathrm{a}$. Les données -4 et $-4,1 \mathrm{~m}$ de la colonne de 
gauche n'ont pas été prises en compte dans cette méthode ni dans la méthode RBF: figure 8-d.

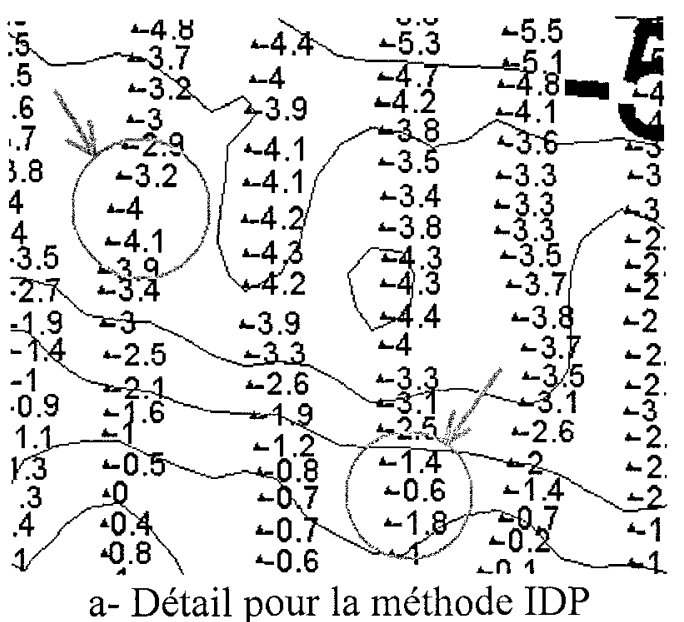

a- IDP method's detail

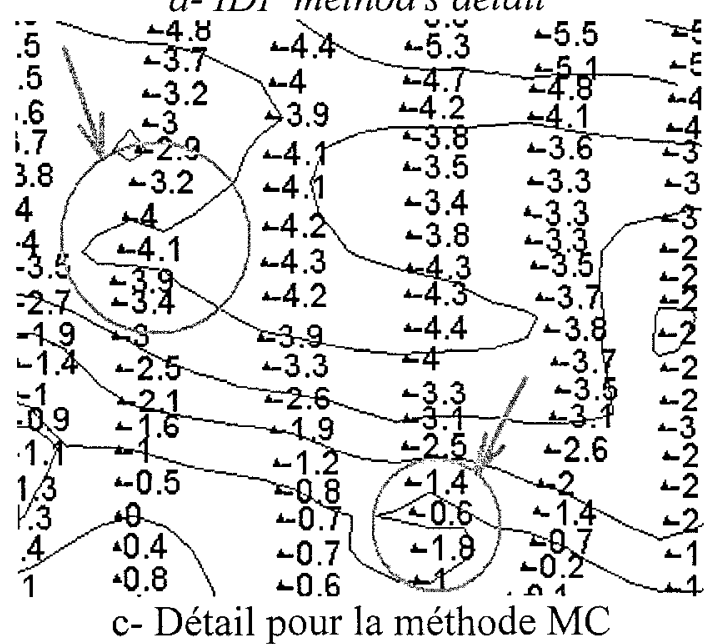

c- MC method's detail

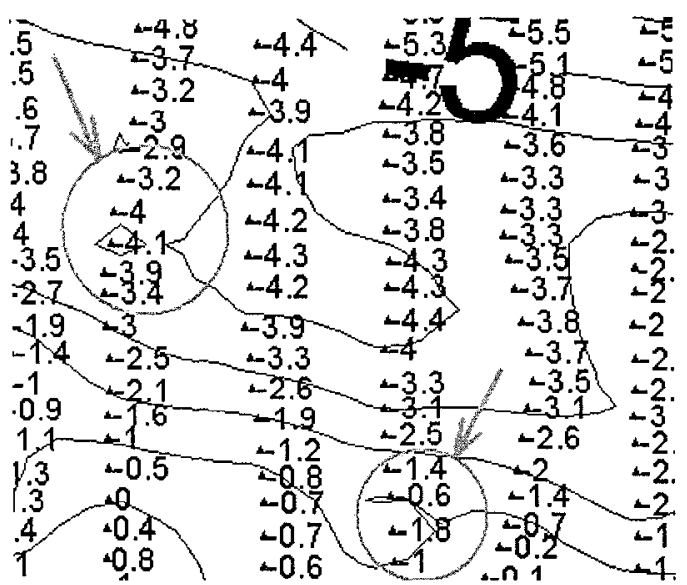

b- Détail pour la méthode $\mathrm{K}$

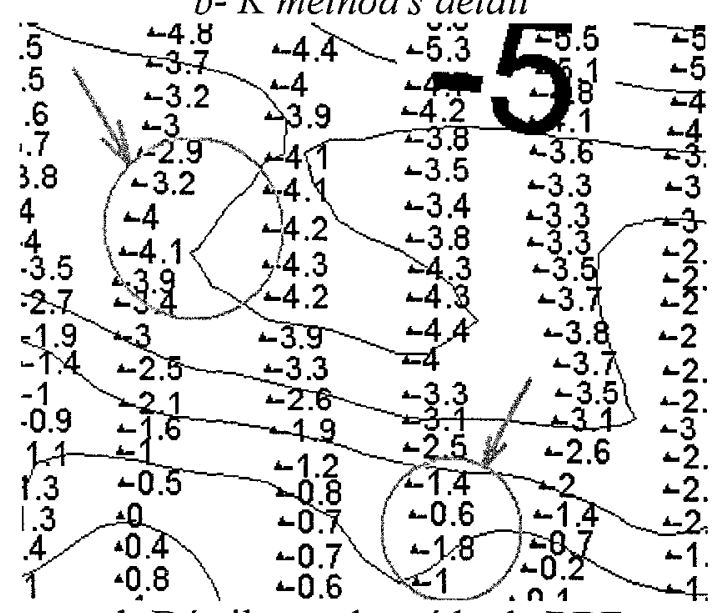

d- Détail pour la méthode RBF

$d$ - RBF method's detail

Fig. 8: Comparaison entre les différentes méthodes - Détails

Fig. 8 : Comparison between the different methods-Details

Les méthodes $\mathrm{K}$ et $\mathrm{MC}$ peuvent être comparées à partir du même détail: les courbes obtenues par la méthode MC sont plus lissées que celles obtenues par la méthode $\mathrm{K}$. L'observation de l'isobathe $-1 \mathrm{~m}$ au niveau des données $-1,4 ;-0,6$; $-1,8$ contenues dans la troisième colonne des figures $8 \mathrm{~b}$ et $8 \mathrm{c}$, montre qu'avec la méthode $\mathrm{K}$ la valeur $-0,6 \mathrm{~m}$ se trouve située entre les isobathes $-1 \mathrm{~m}$ et $-2 \mathrm{~m}$; alors que la méthode $\mathrm{MC}$ l'a correctement représentée.

Cette étude montre que les meilleures méthodes semblent être les méthodes dites "Kriging" et "Minium Curvature". La première répond mieux au calcul des résidus et des volumes mais présente de légères erreurs dans la représentation des isobathes tandis que la seconde obtient de moins bons résultats sur les deux premiers critères mais assure une bonne représentation des fonds. La méthode "Kriging" est proposée par défaut dans "Surfer", ce qui semble être un bon choix. 


\section{Conclusions}

Après avoir obtenu vingt deux cartes de relevés bathymétriques relatant l'historique du site des plages d'Anglet, une analyse de la meilleure méthodologie à suivre, pour numériser ces données afin de les traiter par la suite, a été réalisée. Il a tout d'abord été montré que contrairement à l'idée première, il convient de numériser les points de mesures et non la position des isobathes. Dans un deuxième temps, les importantes variations, engendrées par des points de mesures moins nombreux, ont été mises en évidence. Ceci conduit à numériser tous les points disponibles. Enfin la comparaison des différentes méthodes de modélisation proposées par le logiciel "Surfer", a démontré que les algorithmes "Kriging" et "Minimum Curvature" semblaient donner les meilleurs résultats sur le site d'Anglet.

\section{Remerciements}

Nous tenons à remercier la Communauté d'Agglomérations de Bayonne Anglet Biarritz pour le financement de cette étude, ainsi que la Sogreah et la DDE maritime du port de Bayonne pour l'aide qu'ils nous ont apportée dans la réalisation de cette étude et tout particulièrement pour la mise à disposition des données permettant de réaliser l'historique du site. Cette étude s'inscrit également dans le cadre du PNEC-ART7.

\section{Bibliographie}

[1] Polette R., "Protection des plages contre l'érosion. Evaluation des méthodes de rechargement de l'avant-plage", Rapport de Travaux de Fin d'Etudes ENTPE, 1996, 59 p.

[2] Gorman J., Morang A., Larson R., "Monitoring the coastal environment; Part IV: Mapping, shoreline changes, and bathymetric analysis", Journal of Coastal Research, 14(1), 1998, pp.61-92

[3] Devilliers O., "Improved Incremental randomized Delaunay triangulation", Rapport de recherche, INRIA, nov 1997, 21 p.

[4] Balaven S., Bennis C., Boissonat J.D., Sarda S., "Generation of hybrid grids using power diagrams", $7^{\text {th }}$ International Conference on Numerical Grid Generation in Field Simulations,.Whisthler, Canada, Sept 2000, 10 p.

[5] "Surfer User's guide", 3 édition, Golden Software Inc., 1999, 619 p.

[6] Cressie N.A.C., "Statistics for Spatial Data", John Wiley and Sons Inc.,New York, 1991, 900 p.

[7] Cressie N.A.C., "The Origin of Kriging", Mathematical Geology, 22, 1990 , p. 239-252

[8] Smith W.H.F., Wessel P., "Gridding with continous Curvature Splines In Tension", 55(3), 1990, p.293-305

[9] Davies J.C., "Statistics and Data Analysis in Geology", John Wiley and Sons Inc.,New York, 1986.

[10] Press W.H., Flannery B.P., Teukolsky S.A., Vetterling W.T.,"Numerical Recipes in C", Cambridge University Press., 1988. 